\title{
Análise da funcionalidade de pacientes com hemiparesia pós- AVE por meio do WHODAS 2.0
}

\author{
Functioning analysis of patients with post-stroke hemiparesis using \\ WHODAS 2.0
}

\section{Eduardo Henrique Ferreira ${ }^{1}$, Andrei Pereira Pernambuco ${ }^{2}$, Hugo Augusto Gonsalves da Silva ${ }^{2}$, Pedro Fiche Pereira ${ }^{2}$, Mariana Oliveira Santos ${ }^{2}$, Renata Antunes Lopes ${ }^{2}$, Cláudia Maria Byrro Costa ${ }^{1}$, Carolina Marques Carvalho Mitre Chaves ${ }^{2}$}

${ }^{1}$ Faculdade de Ciências Médicas de Minas Gerais, Programa de Pós-graduação em Fisioterapia Neurológica Adulto e Infantil, Belo Horizonte, Minas Gerais, Brasil.

${ }^{2}$ Universidade de Itaúna, Faculdade de Fisioterapia, Itaúna, Minas Gerais, Brasil.

\section{Resumo}

Introdução: O WHODAS 2.0 (World Health Organization Disability Assessment Schedule) se baseia no conceito principal da CIF (Classificação Internacional de Funcionalidade, Incapacidade e Saúde) e abrange o nível de funcionalidade de um indivíduo em seis grandes domínios da vida. Esse instrumento pode direcionar a conduta dos fisioterapeutas frente às principais dificuldades apresentadas pelos indivíduos com hemiparesia. Objetivo: Analisar a funcionalidade de pacientes com hemiparesia em atendimento fisioterapêutico ambulatorial. Metodologia: Vinte e dois pacientes com hemiparesia que estão em atendimento fisioterapêutico em uma clínica escola participaram do estudo. Os instrumentos de triagem e coleta de dados foram o Mini Exame do Estado Mental, o WHODAS 2.0 administrado por entrevistador e o WHODAS 2.0 administrado ao proxy, ambos de 36 itens. Resultados: Observa-se uma perda funcional desses indivíduos em todos os domínios que compõem o WHODAS 2.0, e o Atividades de vida foi o domínio mais impactado. Com relação aos itens que compõem cada domínio, os que tiveram pior desempenho foram aprender uma nova tarefa, por exemplo, como chegar a um lugar desconhecido; ficar em pé por longos períodos, mais de 30 minutos; ficar sozinho sem a ajuda de outras pessoas por alguns dias; ter atividades sexuais; realizar as atividades diárias do trabalho; tempo gasto com a sua condição de saúde ou suas consequências. Conclusão: A partir dessas informações, programas terapêuticos podem ser aplicados de forma direcionada às necessidades dessa população, assim como recomenda a Organização Mundial da Saúde (OMS).

Palavras-Chave: Fisioterapia; Acidente Vascular Encefálico; Funcionalidade; CIF; Hemiparesia. 


\section{Abstract}

Introduction: The WHODAS 2.0 (World Health Organization Disability Assessment Schedule) is based on the main concept of ICF (International Classification of Functioning, Disability, and Health) and covers the level of functionality of an individual in six major domains of life. This instrument can direct the conduct of physiotherapists in face of the main difficulties presented by individuals with hemiparesis. Objective: To analyze the functionality of patients with hemiparesis in outpatient physiotherapeutic care. Methodology: Twenty-two patients with hemiparesis who are in physiotherapeutic care at a clinical school participated in the study. The instruments for screening and data collection were the Mini Mental State Exam, the WHODAS 2.0 administered by the interviewer, and the WHODAS 2.0 administered to the proxy, both of 36 items. Results: It is observed a functional loss of these individuals in all the domains that make up the WHODAS 2.0, being the Life activities the domain more impacted. With respect to the items that make up each domain, the ones that had worst performance were learning a new task, for example, learning how to get to a new place; standing for long periods such as 30 minutes; staying by yourself for a few days; sexual activities; do your day-to-day work; time spent on your health condition or its consequences. Conclusion: From this information, therapeutic programs can be applied in a way directed to the needs of this population, as recommended by the World Health Organization (WHO).

Keywords: Physiotherapy; Stroke; Functionality; ICF; Hemiparesis.

\section{Introdução}

O Acidente Vascular Encefálico (AVE) é uma afeç̧ão com alta incidência e responsável pela ocorrência de múltiplas e duradouras incapacidades em adultos ${ }^{1}$. O AVE é o segundo maior responsável por mortes em adultos no mundo, ocorrem cerca de 5,7 milhões de casos anuais, o que representa $10 \%$ de todos os óbitos ${ }^{2}$. No Brasil, é considerado a primeira causa de incapacidade entre adultos ${ }^{3}$. O AVE em adultos jovens está cada vez mais frequente $\mathrm{e}$ impacta negativamente na funcionalidade, principalmente ao comprometer a atividade e participação das pessoas acometidas por essa condição $0^{4}$

O AVE provoca a morte de neurônios e, consequentemente, a falta de conexão entre eles, o que resulta em uma comunicação indevida do sistema nervoso central com os seus efetores ${ }^{5}$. Como consequência, o indivíduo poderá apresentar alterações sensório-motoras, comportamentais e/ou cognitivas, as alterações sensóriomotoras mais prevalentes são a hemiplegia ou hemiparesia, espasticidade, encurtamento muscular, alterações de equilíbrio e coordenação motora, que podem levar a limitações de atividade, como incapacidade de executar a marcha, alcance e movimentos finos distais dos membros superiores ${ }^{5}$. No que concerne às alterações comportamentais, o paciente poderá apresentar sinais de depressão, ansiedade e, em casos mais extremos, agressividade $^{6}$. Em relação às alterações cognitivas, o paciente poderá apresentar dificuldades de memória, déficit de atenção e alterações na linguagem. As alterações de estrutura e função corporal associadas às limitações de atividade e restrições na participação social causam um déficit funcional que pode variar de pessoa para pessoa ${ }^{3}$.

A perda da funcionalidade, no entanto, leva a maiores dificuldades na capacidade do indivíduo se inserir ao meio no qual ele vive, o que faz com que ele se torne, inclusive, mais predisposto a outras patologias, tais como as neuropsicopatologias ${ }^{7}$. Frente a esse cenário, faz-se necessária a avalição dos níveis de funcionalidade/incapacidade dessa população. De acordo com a Classificação Internacional de Funcionalidade, Incapacidade e Saúde (CIF), criada em 2001 pela Organização Mundial de Saúde, o termo funcionalidade possui caráter positivo e compreende a integridade das funções e estruturas corporais além de abranger as atividades e participação social do indivíduo ${ }^{8}$. 
A Escala de Avaliação de Incapacidade WHODAS 2.0 (World Health Organization Disability Assessment Schedule) criada pela Organização Mundial de Saúde (OMS) é baseada na $\mathrm{CIF}^{9}$. Esse instrumento se baseia no conceito principal da CIF e abrange o nível de funcionalidade de um indivíduo em seis grandes domínios da vida, são eles, cognição; mobilidade; autocuidado; relações interpessoais; atividades de vida; $\mathrm{e}$ participação ${ }^{9}$. Todos esses domínios foram selecionados a partir de um conjunto de itens que compõem a CIF, todos os itens foram extraídos do componente atividade e participação. Para todos os seis domínios, o WHODAS 2.0 apresenta uma classificação de funcionalidade e incapacidade de confiança e de fácil replicabilidade ${ }^{10}$. Devido a sua fácil utilização, o WHODAS já foi aplicado em populações com diferentes patologias, tais como dores musculoesqueléticas ${ }^{11}$ e doença pulmonar obstrutiva crônica ${ }^{12}$, no entanto, existem poucos estudos utilizando o WHODAS 2.0 para pacientes brasileiros que apresentam alguma doença neurológica, portanto, são necessárias mais pesquisas com foco nesse instrumento.

Assim, diante da importância do entendimento da funcionalidade e para garantir um tratamento mais completo e com base nas necessidades dos pacientes com hemiparesia, este estudo tem por objetivo avaliar as principais incapacidades/limitações apresentadas por uma amostra de pessoas que sofreram um episódio de AVE, por meio da aplicação do WHODAS 2.0.

\section{Método e materiais}

\subsection{Tipo de estudo}

Estudo quantitativo, observacional e transversal.

\subsection{Amostra}

Foram incluídos no estudo indivíduos com hemiparesia pós-AVE recrutados em uma clínica escola de Fisioterapia localizada no interior do estado de Minas Gerais. A amostra foi formada por busca ativa. Nenhum cálculo amostral foi realizado.

\subsection{Cuidados Éticos}

O projeto de pesquisa foi aprovado pelo Comitê de Ética por meio do parecer $n^{\circ} 1.286 .528$. No início da pesquisa, os pacientes foram informados sobre os objetivos, riscos e benefícios decorrentes da participação na pesquisa e os que aceitaram participar do estudo, assinaram um termo de consentimento livre e esclarecido em duas vias. Todos os procedimentos éticos estavam de acordo com a resolução 466/12 do Conselho Nacional de Saúde.

\subsection{Critérios de inclusão e exclusão}

Foram incluídos no estudo, indivíduos com hemiparesia pós-AVE com idade entre 50 e 90 anos; com ou sem déficit cognitivo; de ambos os sexos e que frequentam a clínica escola do curso de Fisioterapia de uma Instituição de Ensino Privada do interior de Minas Gerais. Foram excluídos os pacientes que passaram por cirurgias nos últimos cinco anos; pacientes não residentes no município em que se localiza a clínica; os que possuíam problemas visuais ou auditivos que impedissem a comunicação com os pesquisadores, os com doenças reumatológicas e autoimunes em curso e os que se recusaram a participar da pesquisa como voluntários.

\subsection{Instrumentos}

Como instrumento de coleta de dados foi utilizado o Mini Exame do Estado Mental (MEEM) ${ }^{13}$ e o WHODAS 2.0 administrado por entrevistador e administrado ao proxy, traduzido e validado para a população brasileira por Castro \& Leite, $2015^{14}$.

A presença ou não de déficit cognitivo foi determinada pelos pontos de corte no MEEM, baseado na escolaridade (13 pontos para analfabetos; 18 pontos para pessoas com escolaridade de 1 a 8 anos; 26 para mais de 08 anos), que foram criados por Folstein et al. no ano de $1975^{13}$. Este questionário foi traduzido e validado para a população brasileira por Bertolucci, $1994^{15}$. O MEEM foi utilizado para saber qual seria o questionário ideal do WHODAS 2.0 para cada paciente (administrado por entrevistador ou administrado ao proxy). 
O nível de funcionalidade/incapacidade dos participantes foi avaliado pelo WHODAS 2.0 administrado por entrevistador, classificados por meio dos seis domínios de vida presentes na seção 4 , quais sejam: cognição - compreensão e comunicação; mobilidade movimentação e locomoção; autocuidado - lidar com a própria higiene, vestir-se, comer e permanecer sozinho; relações interpessoais - interações com outras pessoas; atividades de vida - responsabilidades domésticas, lazer, trabalho e escola; participação - participar em atividades comunitárias e na sociedade. Para os pacientes que obtiveram uma pontuação abaixo do ponto de corte no MEEM, foi utilizado o WHODAS 2.0 administrado ao proxy, e algum familiar, amigo ou cuidador foi o responsável em responder o questionário para o paciente ${ }^{9}$. Tanto o WHODAS 2.0 administrado por entrevistador quanto o administrado ao proxy, utilizaram a versão completa a qual contém 36 questões. As questões para cada domínio são respondidas indicando o nível de dificuldade, em uma escala ordinal de cinco níveis "nenhuma" (um), "leve" (dois), "moderada" (três), “grave" (quatro), "extrema ou não consegue fazer" (cinco) - no desempenho ou participação em atividades, considerando o período relativo aos 30 dias anteriores à avaliação $^{14}$.

\subsection{Procedimentos}

A coleta de dados se iniciou com a aplicação do MEEM por um avaliador independente e cegado para os objetivos do estudo, em sequência, o mesmo avaliador aplicou o questionário WHODAS 2.0 ao paciente ou ao seu proxy. Antes da aplicação dos questionários o avaliador foi treinado a fim de melhorar a confiabilidade intraexaminador.

\subsection{Análise dos dados}

A análise dos dados foi feita por meio da estatística descritiva. Medidas de tendência central (média e mediana) e de dispersão (desvio padrão) foram utilizadas. As análises foram realizadas no software Microsoft Excel 2010.

\section{Resultados}

A amostra foi composta por 22 indivíduos que haviam sofrido um episódio de AVE, com idade entre 52 e 86 anos, (64 $\pm 10,08$ anos). O perfil da maioria dos participantes do estudo foi de homens (64\%), casados (64\%), aposentados (77\%), e com baixo nível de escolaridade $(73 \%)$.

A FIGURA 1 apresenta os seis domínios presentes no WHODAS 2.0. É possível observar que o domínio com pior desempenho foi o relacionado às atividades de vida $(3,56 \pm 0,95)$, tais como, cuidar das suas responsabilidades domésticas, do trabalho e escolares. 


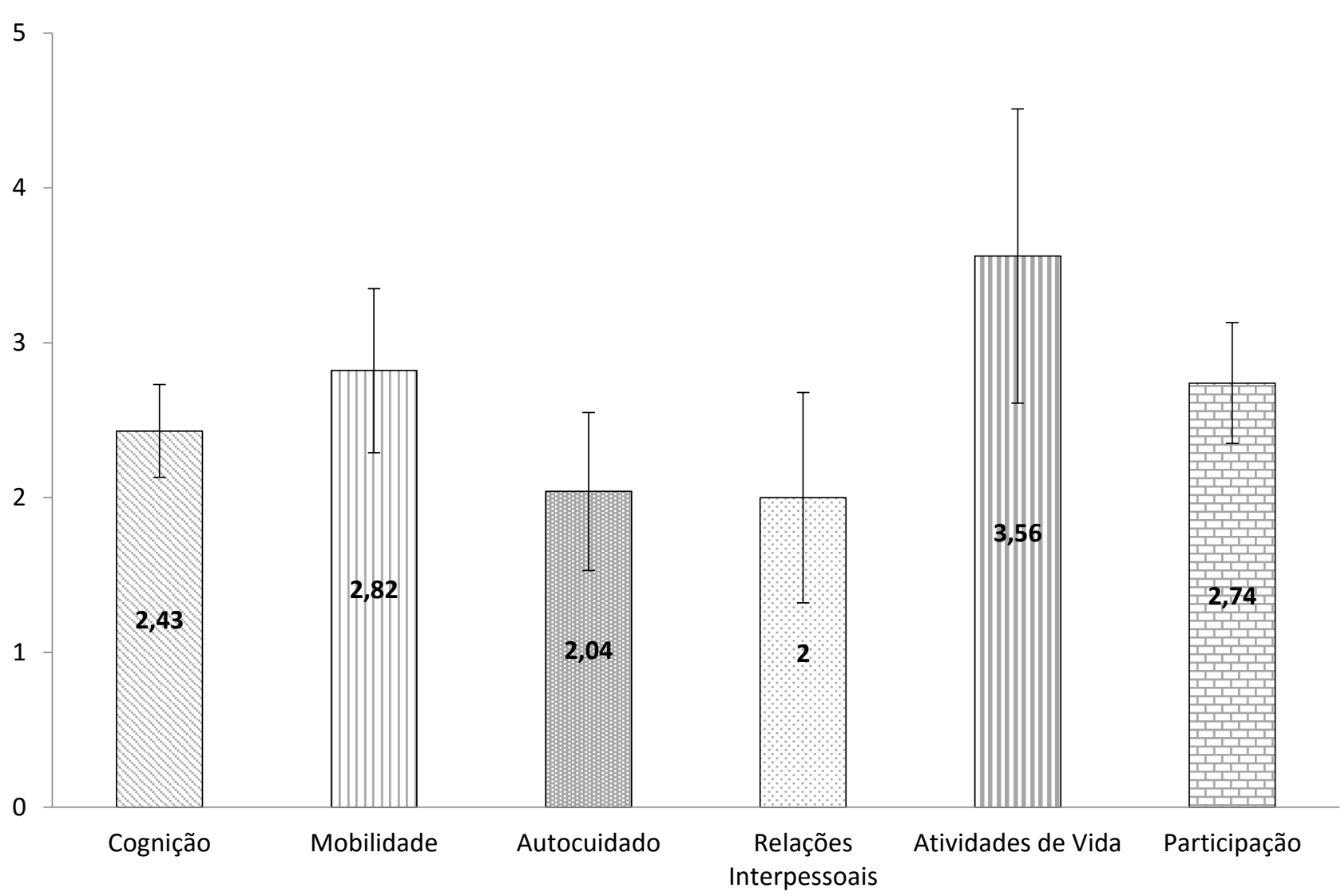

FIGURA 1 - Apresentação dos seis domínios presentes no WHODAS 2.0, indicando domínio com o pior desempenho. As barras representam a média e os traços o desvio padrão.

A FIGURA 2 indica a média e desvio padrão dos itens mais impactados em cada domínio do WHODAS 2.0. Pode-se observar que o componente com o pior desempenho no domínio cognição foi o D1.4 (2,86 $\pm 0,30)$ - aprender uma nova tarefa, por exemplo, como chegar a um lugar desconhecido. A maior parte dos participantes possui dificuldades em realizar essa tarefa.

Com relação ao domínio mobilidade, pode-se observar que o componente com o pior desempenho foi o D2.1 $(3,55 \pm 0,53)$ - ficar em pé por longos períodos como 30 minutos - a maioria dos participantes possuem dificuldades em realizar esta tarefa.

No domínio autocuidado, pode-se observar que o componente com o pior desempenho foi o D3.4 (2,68 \pm $0,51)$ - ficar sozinho sem a ajuda de outras pessoas por alguns dias - esse foi o item mais impactado do domínio autocuidado.
O componente do domínio relações interpessoais com o pior desempenho foi o D4.5 $(3,36 \pm 0,68)-$ ter atividades sexuais - a maioria dos participantes relata dificuldades em realizar essa tarefa.

Com relação ao domínio atividades de vida, é possível observar que o componente com o pior desempenho foi o D5.5 $(4,64 \pm 0,95)$ - realizar as atividades diárias do trabalho, no qual maioria dos participantes possuem dificuldades em realizar esta tarefa.

Com relação ao domínio participação, observase que o componente com o pior desempenho foi o D6.4 $(3,36 \pm 0,39)$ - o tempo gasto com a sua condição de saúde ou suas consequências - item mais impactado do domínio participação. 


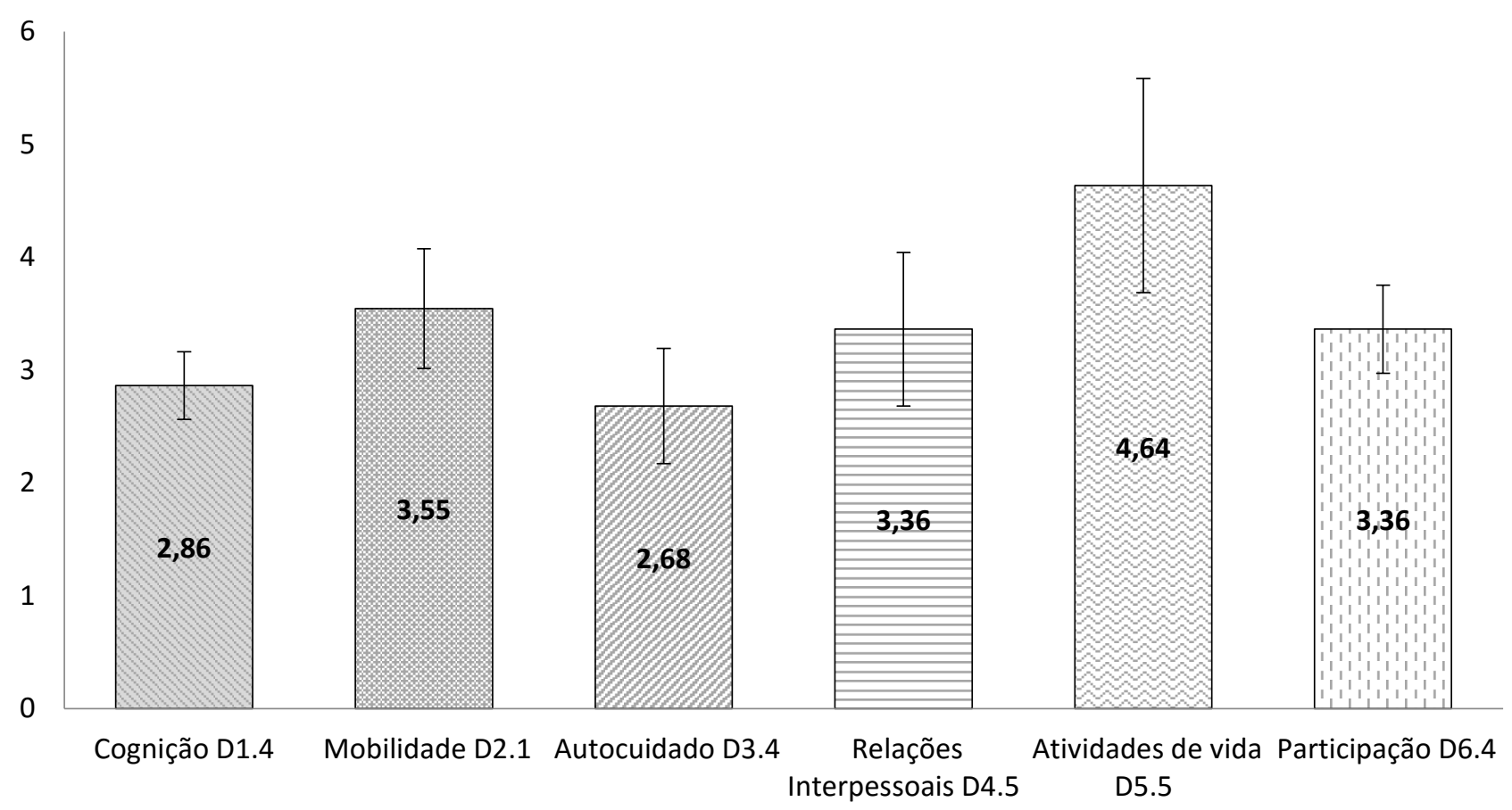

FIGURA 2 - Apresentação dos componentes mais impactados presentes no WHODAS 2.0, indicando o item com o pior desempenho em cada domínio. As barras representam a média e os traços o desvio padrão.

\section{Discussão}

No presente estudo foi possível demonstrar que os indivíduos com hemiparesia pós-AVE atendidos em uma clínica escola de fisioterapia do interior de Minas Gerais apresentaram considerável perda no nível de funcionalidade, com impacto negativo em todos os domínios que compõem o WHODAS 2.0. O conhecimento do perfil funcional da população com hemiparesia pós-AVE pode contribuir para que as reais condições de vida dessas pessoas façam parte das estatísticas, permitindo delinear políticas públicas, definir intervenções e destinar orçamentos ${ }^{16}$.

A amostragem total do estudo foi de 22 indivíduos com hemiparesia, $64 \%$ da amostra foi composta por homens e $36 \%$ da amostra foi composta por mulheres. A predominância do AVE em indivíduos do sexo masculino corrobora os achados da literatura ${ }^{17}$.

Devido à alta incidência do AVE e, as consequências dessa condição, é necessário que os indivíduos acometidos se beneficiem de programas de intervenção funcional adequados ao seu perfil funcional e variabilidade individual $^{18}$. No entanto, para tal, faz-se necessária uma avaliação global do paciente com o intuito de identificar as principais dificuldades apresentadas por esta população, a fim de potencializar o efeito do tratamento $^{19}$.

Neste trabalho, para mensuração da funcionalidade, foi utilizado o WHODAS 2.0, que é considerado uma ferramenta de grande potencial e com validade testada para o processo de identificação das principais incapacidades desenvolvidas por indivíduos com diferentes condições de saúde, esse instrumento pode fornecer dados padronizados sobre a saúde e os aspectos relacionados à saúde de indivíduos e ou populações $\operatorname{distintas}^{14}$.

Dentre os domínios avaliados pelo WHODAS 2.0, o domínio com pior desempenho foi o relacionado às atividades de vida, tais como, cuidar das suas responsabilidades domésticas (categorias CIF: d630 d649), do trabalho e escolares. Esse item pode ser justificado pelo fato de os indivíduos, após um episódio 
de AVE, apresentarem alterações em várias funções corporais, dentre elas, a diminuição da força muscular no hemicorpo contralateral a lesão encefálica, conforme discutido por Langhorne et al ${ }^{20}$. Já Maeshima et al. (2013) indicam que indivíduos pós-AVE, principalmente na fase aguda, necessitam de um apoio familiar muito grande para ajudar nas atividades de vida, o que justifica a sua dificuldade em retornar às atividades do trabalho, escolares e domésticas ${ }^{21}$.

A dificuldade apresentada pelos participantes na questão "aprender uma nova tarefa, por exemplo, como chegar a um lugar desconhecido" (categoria CIF: d2101) que integra o domínio Cognição, pode ser justificada, pelo menos em parte, pelo fato de que indivíduos com hemiparesia podem apresentar sinais de alterações cognitivas, dependendo do local da lesão, idade do indivíduo, nível educacional, dentre outros motivos, o que corrobora com os achados da literatura ${ }^{22}$. Outro motivo pode ser a falta de motivação em realizar novas tarefas devido ao seu estado atual de saúde. De acordo com Hackett et al. (2005), a maioria dos portadores de AVE desenvolvem depressão, devido a uma instabilidade da labilidade emocional do mesmo ${ }^{23}$

Com relação ao domínio Mobilidade, a atividade mais impactada foi a de ficar em pé por longos períodos como 30 minutos (categoria CIF: d4154), o que pode ser justificado pela deficiência do controle postural presente nesses indivíduos ${ }^{24}$. Uma vez que o controle postural é a capacidade do ser humano de manter-se ereto ou executar movimentos sem oscilação ou queda, é compreensível os motivos pelo qual os indivíduos com hemiparesia apresentam dificuldade em realizar diversas tarefas motoras, tais como, ficar de pé, alcançar objetos, levantar de uma cadeira e caminhar. Normalmente, o controle postural é garantido pela interação entre o sistema sensorial, motor e cognitivo, os quais vão resultar na estabilidade entre os segmentos corporais, aferência e processamento das informações sensoriais eficientes e a resposta adequada do sistema musculoesquelético ${ }^{24}$. Em pessoas que sofreram episódio de AVE, todas as funções corporais descritas acima podem estar prejudicadas e podem resultar em anormalidades tanto na orientação/alinhamento postural quanto no equilíbrio das forças que agem sobre o corpo ${ }^{25}$. Nesse contexto, um dos principais objetivos da fisioterapia é tratar essa deficiência, que é fundamental para as inúmeras tarefas funcionais, como por exemplo, a marcha, tornando o indivíduo capaz de executar as suas atividades de vida diária de forma natural e precisa ${ }^{20}$.

Com relação ao domínio de Autocuidado, “a dificuldade de ficar sozinho sem a ajuda de outras pessoas por alguns dias" (categoria CIF: d5702) pode estar relacionada à necessidade de auxílio, principalmente na fase aguda da doença. De acordo com a literatura ${ }^{26}$, os cuidadores familiares possuem diferentes necessidades em diferentes estágios da doença, é necessária uma bagagem de conhecimento sobre as mudanças na condição de saúde do paciente. Os cuidadores esperam que os profissionais da área da saúde respondam com honestidade às perguntas sobre a condição atual do paciente e possíveis mudanças. Se tal informação não é fornecida aos cuidadores no estágio agudo, pode resultar em incerteza e ansiedade, o que pode dificultar a relação entre cuidadores e pacientes ${ }^{26}$. Outro estudo reforça a importância dos familiares no processo de reabilitação desses pacientes, ou seja, os familiares também possuem um papel importante no processo da reintegração social destes, além de lhes proporcionar um maior bem-estar e confiança $^{21}$.

No domínio Relações Interpessoais, o componente mais impactado foi "ter atividades sexuais" (categoria CIF: d7702), em que os participantes relataram não realizar a tarefa ou ter uma dificuldade extrema. Segundo a literatura ${ }^{27}$, o AVE pode proporcionar alterações sensoriais no lado corporal contralateral à lesão encefálica, o que pode diminuir a sensação de prazer desses indivíduos. Além disso, ocorrem também mudanças físicas, tais como diminuição do condicionamento cardiovascular ${ }^{28}$, e, em alguns casos, labilidade emocional ${ }^{22}$, fazendo com que percam a libido e desistam de realizar tal atividade. No entanto, essa questão deve ser desmitificada principalmente pelos 
fisioterapeutas, que devem estar preparados para abordála tanto na avaliação do paciente quanto no processo terapêutico.

Dentro do domínio Atividades de vida, o componente mais impactado foi "realizar as atividades do trabalho/escola" (categoria CIF: d840 - d859). Sabe-se que os indivíduos com hemiparesia apresentam comprometimento do membro superior e inferior, o que os impossibilita de realizar certas funções tanto na fase aguda quanto crônica da doença ${ }^{20}$, como os movimentos finos com as mãos e ficar em pé por longos períodos, conforme já discutido. Além disso, esses fatores podem contribuir para um isolamento social, e levar o indivíduo a se sentir incapaz de trabalhar novamente ${ }^{29}$. E é justamente nesse momento que o apoio familiar será de grande valia para restabelecer a segurança e aumentar a autoestima desses indivíduos ${ }^{26}$. Além do apoio familiar, atualmente, a fisioterapia neurofuncional abrange um modelo teórico por meio da abordagem sistêmica do indivíduo, na qual o foco do tratamento também se dá na tarefa realizada e no ambiente no qual ele está inserido, considerando-o como um ser biopsicossocial. Uma forma de abranger o indivíduo de forma completa é levar em consideração os 4 "P" da fisioterapia, prevenção, previsão, plasticidade e participação ${ }^{30}$. Assim, o terapeuta terá um olhar da real condição do paciente e das principais intervenções a serem realizadas, o que garantirá a este uma melhor qualidade de vida.

O último domínio avaliado foi o de Participação, "tempo gasto com a sua condição de saúde ou suas consequências" (categoria CIF: d910) este foi o mais impactado. Nesse item, os participantes relataram ter gasto mais tempo cuidando da saúde do que realizando atividades prazerosas com a família, o que pode ser justificado pelo fato do AVE ser uma afecção limitante e que exige cuidados, principalmente na fase aguda e crônica, e, na maioria das vezes, os responsáveis por cuidar desses indivíduos são os familiares ${ }^{31}$. Assim, fazse necessária a compreensão dos familiares do quadro do paciente, não exigindo dele coisas além da sua capacidade atual, evitando assim que se sinta frustrado com os resultados.

Os achados deste estudo são importantes, pois demonstram como o Acidente Vascular Encefálico interfere nos níveis de funcionalidade de um indivíduo, o que permite que os profissionais da saúde o entendam como um ser biopsicossocial. Com esses achados é importante ter como metas de tratamento para essa população o treino de Atividades de vida diárias (AVDs), a melhora do condicionamento físico, o treino de equilíbrio e propriocepção, o fortalecimento muscular aliado a exercícios para o aumento da amplitude de movimento, além de abordar a vida sexual desse indivíduo com a (o) sua (seu) parceira (o), com o intuito de ajudá-lo (a) no que for possível.

As principais limitações do estudo são: o pequeno tamanho amostral que impede que os resultados sejam generalizados e; o desenho transversal do estudo, que não permite o acompanhamento da situação do participante por um período prolongado de tempo. Novos estudos que visem esclarecer as inconsistências aqui apresentadas devem ser realizados.

\section{Conclusão}

Foi possível observar uma perda funcional desses indivíduos em todos os domínios que compõem o WHODAS 2.0, o que indica a grande necessidade da atuação do fisioterapeuta durante a instalação dessa doença.

As principais dificuldades apresentadas por indivíduos com hemiparesia em cada domínio do WHODAS 2.0 foram: aprender uma nova tarefa (domínio cognição), ficar em pé por longos períodos como 30 minutos (domínio mobilidade); ficar sozinho sem a ajuda de outras pessoas por alguns dias (domínio autocuidado); ter atividades sexuais (domínio relações interpessoais); realizar as atividades do trabalho/escola (domínio atividades de vida); tempo gasto com a sua condição de saúde ou suas consequências (domínio participação). O 
domínio mais impactado neste trabalho foi atividades de vida, o que indica, mais uma vez, que o AVE é uma doença incapacitante e que precisa da intervenção fisioterapêutica desde o início da sua instalação.

Os achados desse estudo devem direcionar os fisioterapeutas nas intervenções clínicas, podendo ter como objetivo não apenas tratar estas incapacidades, mas, principalmente, prevenir que as mesmas se instalem precocemente. Sugere-se ainda a realização de novos estudos com um tamanho amostral maior para a obtenção de maiores resultados.

\section{Declaração de conflitos de interesse}

Os autores do artigo afirmam que não houve nenhuma situação de conflito de interesse, tais como propostas de financiamento, emissão de pareceres, promoções ou participação em comitês consultivos ou diretivos, entre outras, que pudessem influenciar no desenvolvimento do trabalho.

\section{Referências}

1.

FRENCH, B.; THOMAS, L.H.; COUPE, J.; et

al. Does Repetitive Task Training Improve Functional Activity After Stroke? A Cochrane Systematic

Review and Meta-Analysis. Revista Cochrane Database of Systematic Reviews, vol. 11, Art. No.: CD006073, 2016.

2. BOTELHO, T.S.; NETO, C.D.M.; ARAÚJO, F.L.C.; ASSIS, S.C. Epidemiologia do acidente vascular cerebral no Brasil. Revista Temas em Saúde, João Pessoa, vol.16, n.2, 2016.

3. MARTINS, S.C.O.; NETO, O.M.; ALVES, C.V.; FREITAS, G.R.; et.al. Past, present, and future of stroke in middle-income countries: the Brazilian experience. Revista International Journal of Stroke, 2013.

4. CABRAL, N.L.: FREIRE, A.T.: CONFORTO, A.B.: SANTOS, N.: REIS, F.I.: NAGEL, V.: et al. Increase of Stroke Incidence in Young Adults in a
Middle-Income Country: A 10-Year Population-

Based Study. Revista American Heart Association, DOI: 10.1161/STROKEAHA.117.018531, 2017.

5. MOCHIZUKI, L.; BIGONGIARI, A.; FRANCIULLI, P.M.; et al. The effect of gait training and exercise programs on gait and balance in poststroke patients. Revista Medical Express, São Paulo, vol. 2, 2015.

6. KIM, J.S.; CHOI, S.; KWON, S.U.; SEO, Y.N. ; Inability to control anger or aggression after stroke. Revista American Academy of Neurology, vol. 58(7), 2002.

7. VALE, T.C.C.; LIMA, A.D.; FERREIRA, E.H.; GUIMARÃES, R.A.S.; COELHO, M.A.G.M.;

PERNAMBUCO, A.P. Principais limitações na atividade e restrições na participação apresentadas por idosos sob a perspectiva de um Core Set da CIF. Revista Conexão Ciência, Formiga/MG, vol. 12, n. 2, p.99-109, 2017.

8. Classificação Internacional de Funcionalidade, Incapacidade e Saúde. Centro colaborador da Organização Mundial da Saúde para família de classificações internacionais em português. 1 . Ed. São Paulo: Editora da Universidade de São Paulo, 2015.

9. SILVEIRA, C.; PARPINELLI, M.A.; PACAGNELLA, R.C.; et al. Adaptação transcultural da Escala de Avaliação de Incapacidades da Organização Mundial de Saúde (WHODAS 2.0) para o Português. Revista da Associação Médica Brasileira, Campinas/SP, vol. 59(3), p. 234-240, 2013.

10. ÜSTÜN, T.B.; CHATTERJI, S.; KOSTANJSEK, N.; REHM, J.; KENNEDY, C.; EPPING-JORDAN, J.; et al., Developing the World Health Organization Disability Assessment Schedule 2.0. Revista Bulletin of the World Health Organization, vol. 88, n. 11, p. 797-876, 2010.

11. SILVA, C.; COLETA, I.; SILVA, A.G.; et al. Adaptação e validação do WHODAS 2.0 em utentes com dor musculoesquelética. Revista Saúde Pública, vol. 47(4), p. 752-8, 2013.

12. SILVA, C.M.S.; NETO, A.C.P.; JÚNIOR, B.R.V.N.; TEIXEIRA, H.P.; SILVEIRA, C.D.; 
MACHADO, A.S.; Incapacidade funcional de pacientes com doença pulmonar obstrutiva crônica através da WHODAS. Revista Acta Fisiátrica, vol. 23(3), p. 125-129, 2016.

13. FOLSTEIN, M.F.; FOLSTEIN, S.E.; MCHUGH, P.R. "Mini-mental state". A practical method for grading the cognitive state of patients for the clinician. Revista Psychiatric Research, vol.12, p.189-198, 1975.

14. CASTRO, S.S.; LEITE, C.F.; Avaliação de Saúde e Deficiência: Manual do WHO Disability Assessment Schedule (WHODAS 2.0). Uberaba: Universidade Federal do Triângulo Mineiro. 2015.

15. BERTOLUCCI, P.H.F.; BRUCKI, S.M.D.; CAMPACCI, S.R.; JULIANO, Y.; The Mini-Mental State Examination in a general population: impact of educational status. Rev. Arq. Neuropsiquiatr. Vol. 52(1), p. 1-7, 1994.

16. NUBILA, H.B, BUCHALLA, C.M.; O papel das Classificações da OMS - CID e CIF nas definições de deficiência e incapacidade. Rev. Bras. Epidemiol, vol. 11, p. 324-35, 2008.

17. APPELROS, P.; STEGMAYR, B.; TERÉNT, A.; Sex differences in stroke epidemiology: a systematic review. Revista American Heart Association, vol. 40, p. 1082 - 1090, 2008.

18. YELNIK, A.P.; BONAN, I.V.; SIMON, O.; GELLEZ-LEMAN, M.C.; Rehabilitation of balance after stroke with multisensorial training: a singleblind randomized controlled study. Revista Neurology, vol. 8, p. 1-15, 2008.

19. FENS, M.; BEUSMANS, G.; LIMBURG, M.; HOEF, L.V.; HAASTREGT, J.V.; METSEMAKERS, J.; et al.; A process evaluation of a stroke-specific followup care model for stroke patients and caregivers; a longitudinal study. Revista BMC Nursing, p. 1-10, 2015.

20. LANGHORNE, P.; COUPAR, F.; POLLOCK, A.; Motor recovery after stroke: a systematic review. Revista Neurology, p. 741-754, vol. 8, 2009.

21. MAESHIMA, S.; Family Support in Stroke
Rehabilitation. Revista International Journal of Physical Medicine and Rehabilitation, vol. 1, 2013.

22. RENJEN, P.; GAUBA, C.; CHAUDHARI, D.; Cognitive Impairment After Stroke. Revista Cureus, p. 1-9, vol. 7(9), 2015.

23. HACKETT, M.L.; HONS, M.A.; YAPA, C.; PARAG, V.; HONS, M.S.; ANDERSON, C.S. et al. Frequency of depression after stroke: a systematic review of observational studies. Revista American Heart Association, p. 1330-1340, 2005.

24. HORAK, F.B.; HENRY, S.M.; SHUMWAYCOOK, A.; Postural perturbations: new insights for treatment of balance disorders. Revista Phys. Ther, $p$. 517-33, vol. 77(5), 1997.

25. MARIGOLD, D.S.; ENG, J.J.; TOKUNO, C.D.; DONNELLY, C.A.;

Contribution of muscle strength and integration of afferent stimulus for postural instability in people with stroke, Revista Neurorehabil. Neural Repair, p. 222-9, vol. 18(4), 2004.

26. TSAI, P.C.; YIP, P.K.; TAI, J.J.; LOU, M.F.; Needs of family caregivers of stroke patients: a longitudinal study of caregivers' perspectives. Revista Dove Medical Press, p.449-457, vol. 3,0, 2015.

27. SCALHA, T.B.; MIYASAKI, E.; LIMA, N.M.; BORGES, G.; Correlações entre funções motoras e sensoriais em membros superiores de hemiparéticos crônicos após AVE. Revista Arq. Neuropsiquiatria, vol. 4, p. 624-629, 2011.

28. COLLE, F.; BONAN, I.; GELLEZ LEMAN, M.C.; BRADAI, N.; YELNIK, A.; Fatigue après accident vasculaire cérébral. Revista Annales de Réadaptation et de médecine physique, vol. 49, p. 361364, 2006.

29. HAUN, J.; RITTMAN, M.; SBERNA, M.; The continuum of connectedness and social isolation during post stroke recovery. Revista Journal of Aging Studies, vol. 22, p. 54-56, 2008.

30. HARRIS, S.R.; WINSTEIN, C.J.; The Past, Present, and Future of Neurorehabilitation: From NUSTEP Through IV STEP and Beyond. Revista Pediatr. Phys. Ther, vol. 29, 2017. 
31. URIMUBENSHI, G.; Activity limitations and participation restrictions experienced by people with stroke in Musanze district in Rwanda. Revista African Health Sciences, vol. 15, p. 917-924, 2015. 